\title{
Some Results on Fuzzy Topological Modules and Fuzzy Topological Submodules
}

\author{
Mohammed M.Ali Al-Shamiri ${ }^{1,2}$ \\ ${ }^{1}$ Department of Mathematics, Faculty of science and arts ,Muhayl Asser \\ King Khalid University, K.S.A. \\ ${ }^{2}$ Department of Mathematics and computer, Faculty of science \\ Ibb University ,ibb,Yemen. \\ Email: mal-shamiri [AT] kku.edu.sa
}

\begin{abstract}
In this paper, we bring together the structure of fuzzy topological space, fuzzy module and that of fuzzy ring to form a combined structure, that of a fuzzy topological R-module. Properties of fuzzy topological R-module ,topological R-submodule and its Properties are also briefly examined. we proved many theorems and corollaries as results shown in this paper.
\end{abstract}

Keywords----Topological module , Fuzzy topological module, Fuzzy topological R- submodule

\section{INTRODUCTION}

As is known, a crisp set is defined by dividing individuals into two types - members and non-members. One notices a distinction between members and non-members of the class represented in a crisp set . But many of the sets and categories we normally use do not show this feature. Its borders seem ambiguous, and moving from one member to another seems gradual and not surprising. This is called a fuzzy set that offers ambiguity by eliminating the boundary between members of the acute class of non-members. In our lives more cases are often not clear and deterministic and cannot be accurately described. Such cases are characterized by ambiguity or inaccuracy that cannot be answered only by yes or no. Lutfi Zadeh [8] in 1965 presented the idea of a fuzzy set to describe mathematically in highly abstract and solving these problems gave a certain degree of membership to each member of a given set. This is actually the basic setting of the mysterious fuzzy set theory. Specify a fuzzy set as a generalization for the characteristic function of the set where the degree of element membership is more general than "Yes" or "No".

A mathematically fuzzy set can be determined by assigning to each potential individual in the universe of discourse a value that represents its grade of membership in the fuzzy set. This grade corresponds to the degree of compatibility of this individual with the concept represented by the fuzzy set . Membership scores are often represented by a number of real values ranging in the closed interval period between 0 and 1 . The concept of a mysterious group was applied in algebra as one of the first branches of the various branches of pure mathematics. The first paper on fuzzy group was published by A. Rosenfeld [7] in 1971, where the concepts of fuzzy subgroups and fuzzy subgroupoid were introduced.In this paper our aim to introduce new notion which extend to the notion of fuzzy topological group and topological ring, this notion different almost on the last, that it needs some additional conditions, more than that, there is many branches of topological module not studied as fuzzy case until now ,because the notion is complex and difficult, here we started the work and we full of hope to continue in this field and the interested from mathematicians.

\section{PRELIMINARIES}

We begin with a few basic definitions and results as our prerequisites.

Definition2.1 [7] Let X be a non empty set and I be the closed interval [0,1]. A fuzzy set $\mu$ on Xis a function on X into Iand the collection of all fuzzy sets on $X$ is denoted by $I^{X}$.The support of a fuzzy set $\mu$, denoted by supp $\mu$, is the crisp set $\left\{\mathrm{x} \in \mathrm{X}: \mu(\mathrm{x})>0\right.$ \}.A fuzzy set with a singleton as its support is called a fuzzy point, denoted by $\mathrm{x}_{\mathrm{t}}$, and defined as,

$$
x_{t}(z)= \begin{cases}t, & \text { for } z=x \\ 0 & \text { otherwise. }\end{cases}
$$

Here we note that a fuzzy singleton $x_{t}$ belongs to a fuzzy set $\mu\left(x_{t} \in \mu\right)$ iff $t \leq \mu(x)$.

Definition2.2 [8] Let $X$ and $Y$ be two nonempty sets and $f: X \rightarrow Y$. If $\lambda$ and $\mu$ are fuzzy sets on $X$ and $Y$ respectively then $f(\lambda)$ and $f^{-1}(\mu)$ are fuzzy sets on $Y$ and $X$ respectively, given by 
and

$$
\mathrm{f}(\lambda)(\mathrm{y})=\left\{\begin{array}{rr}
\sup \left\{\lambda(\mathrm{x}): \mathrm{x} \in \mathrm{f}^{-1}(\mathrm{y})\right\}, \mathrm{if}^{-1}(\mathrm{y}) \neq \phi \\
0, & \text { otherwise }
\end{array}\right.
$$

$$
\mathrm{f}^{-1}(\mu)(\mathrm{x})=\mu(\mathrm{f}(\mathrm{x}))
$$

Definition2.3:[6] Let $X$ be a set and $\tau$ a family of fuzzy subsets of $X$.Then $\tau$ is called fuzzy topology on $X$ if satisfies the following conditions:

(1) $1_{\phi}, 1_{X} \in \tau$.

(2) If $\lambda, \mu \in \tau$, then $\lambda \wedge \mu \in \tau$.

(3) If $\lambda_{i} \in \tau$,for each $i \in \Lambda$, then $\bigvee \lambda_{i} \in \tau$.

The ordered pair $(\mathrm{X}, \tau)$ is called a fuzzy topological space (FTS, for short).Moreover ,the members of $\tau$ is said to be the fuzzy open sets and their complements are said to be fuzzy closed sets.

Definition 2.4[6]: A fuzzy set $\lambda$ in a FTS X is called a nbd. of a fuzzy point $x_{t}$ if and only if there exists a fuzzy open set $\mu$ in $\mathrm{X}$ such that $\mathrm{x}_{\mathrm{t}} \in \mu \leq \lambda$

A nbd.$\lambda$ is said to be fuzzy open if and only if $\lambda$ itself is fuzzy open A fuzzy point $x_{t} \in \bar{A}$ if each neighborhood of $x_{t}$ intersection with $\mathrm{A}$.

Definition2.5 [2] Let $(G, \delta)$ be a fuzzy topological space. The induced topology $\tau_{\delta}$ on the collection $\vartheta(G)$ of all fuzzy singletons of $G$ is defined as the topology generated by $\sigma=\left\{V_{\mu} \mid \mu \in \sigma\right\}$, where $V_{\mu}=\{p \in \vartheta(G) \mid p \in \mu\}$ and hence $\left(\vartheta(G), \tau_{\delta}\right)$ is called an induced topological space.

Definition2.6[4]: Let $f$ be a function from a FTS $X$ to a FTS $Y$. Then $f$ is fuzzy continuous iff $f^{-1}(\mu)$ is fuzzy open in $X$ for each fuzzy open set $\mu$ in $Y$. This definition equivalents the definition bellow.

Definition 2.7[4]: Let $f$ be a function from a FTS $X$ to a FTS $Y$. Then $f$ is fuzzy continuous iff foreach fuzzy point $x_{t}$ in $X$ and each nbd. $\lambda$ of $f\left(x_{t}\right)$ in $Y$, there exists a nbd $\mu$ of $x_{t}$ in $X$ such that $f(\mu) \leq \lambda$

Definition2.8[6]: A function $\mathrm{f}: \mathrm{X} \rightarrow \mathrm{Y}$ is called a fuzzy homeomorphism if $\mathrm{f}$ is bijective, fuzzy continuous and $\mathrm{f}^{-1}$ is fuzzy continuous

Definition2.9[6]: The function $f:(X, T) \rightarrow(Y, U)$ is called fuzzy continuous if, for every $B \in U, f^{-1}(B) \in T$. The function $\mathrm{f}:(\mathrm{X}, \mathrm{T}) \rightarrow(\mathrm{Y}, \mathrm{U})$ is called fuzzy homeomorphism if $\mathrm{f}:(\mathrm{X}, \mathrm{T}) \rightarrow(\mathrm{Y}, \mathrm{U})$ is a bijection and both $\mathrm{f}$ and $\mathrm{f}^{-1}$ are fuzzy continuous.

Definition2.10[8]: A fuzzy subset $\mu$ of a group $G$ is called a fuzzy subgroup of

$\mathrm{G}$ if, for all $\mathrm{x}, \mathrm{y} \in \mathrm{G}$, the following conditions are satisfied:

(i) $\mu(x y) \geq \min (\mu(x), \mu(y))$; and

(ii) $\mu\left(x^{-1}\right) \geq \mu(x)$.

Definition 2.11[5]: Let $(R,+, \cdot)$ be a ring and $\mu$ be a fuzzy set in $R$, then $\mu$ is called fuzzy ring in a ring $(R,+, \cdot)$ if for each $\mathrm{x}, \mathrm{y} \in \mathrm{R}$ :

(1) $\mu(x+y) \geq \min \{\mu(x), \mu(y)\}$.

(2) $\mu(x)=\mu\left(x^{-1}\right)$.

(3) $\mu(x \cdot y) \geq \min \{\mu(x), \mu(y)\}$.

A fuzzy subring of a fuzzy ring $\mu$ is a fuzzy ring $\lambda$ satisfing

$\lambda(\mathrm{x}) \leq \mu(\mathrm{x}) \forall \mathrm{x} \in \mathrm{R}$

Definition2.12 [9,10]:Let $\mathrm{R}$ be a ring and let $\mathrm{M}$ be a leftR-module.A fuzzy set $\mu$ in $\mathrm{M}$ is called a fuzzy left R-module if for each $\mathrm{x}, \mathrm{y} \in \mathrm{M}$ and $\mathrm{r} \in \mathrm{R}$ :

(1) $\mu(x+y) \geq \min \{\mu(x), \mu(y)\}$.

(2) $\mu(x)=\mu\left(x^{-1}\right)$.

(3) $\mu(\mathrm{rx}) \geq \mu(\mathrm{x})$.

(4) $\mu(0)=1$.

Definition 2.13 [5]: Let $X$ be a group and $U, V$ two fuzzy sets in $X$. We define $U V$ and $V^{-1}$ by therespective formula $\mathrm{UV}(\mathrm{x})=\sup _{\mathrm{x}_{1} \mathrm{x}_{2}=\mathrm{x}} \min \left(\mathrm{U}\left(\mathrm{x}_{1}\right), \mathrm{V}\left(\mathrm{x}_{2}\right)\right)$ and $\mathrm{V}^{-1}(\mathrm{x})=\mathrm{V}\left(\mathrm{x}^{-1}\right)$ for $\mathrm{x} \in \mathrm{X}$.

Definition [3-5] Let $X$ be a group and let $(X, \tau)$ be a fuzzy topological space (It is not need that $\tau$ contains all constant fuzzy sets). Then $(X, \tau)$ is a fuzzy topological group if it satisfies the following conditions:

(1) The mapping $f:(X, \tau) \times(X, \tau) \rightarrow(X, \tau)$ defined by $f((x, y))=x y$ is fuzzycontinuous.

(2) The mapping $g:(X, \tau) \rightarrow(X, \tau)$ defined by $g(x)=x^{-1}$ is fuzzy continuous. This definition equivalents the definition bellow.

Definition Let X be a group and let $(\mathrm{X}, \mathrm{T})$ be an FTS. $(\mathrm{X}, \mathrm{T})$ is called a fuzzy topological group or FTG for short if: 
(1) For all $a, b \in X$ and any neighborhood $W$ of fuzzy point $(a b)_{t}$, there are neighborhoods $U$ of $a_{t}$ and $V$ of $b_{t}$ such that $\mathrm{UV} \subseteq \mathrm{W}$.

(2) For all $\mathrm{a} \in \mathrm{X}$ and any neighborhood $\mathrm{V}$ of $\mathrm{a}_{\mathrm{t}}^{-1}$, there exists a neighborhood $\mathrm{U}$ of $\mathrm{a}_{\mathrm{t}}$ such that $\mathrm{U}^{-1} \subseteq \mathrm{V}$.

Definition2.14 [1]: A collection $\omega$ of fuzzy nbds. of $x_{t}$, for $0<t \leq 1$, is called a fundamental system of fuzzy nbds. of $x_{t}$ iff for any fuzzy nbd $\mathrm{V}$ of $x_{t}$, there exists $U \in \omega$ such that $x_{t} \leq U \leq V$.

\section{MAIN RESULTS}

We starting our work by definition of fuzzy topological R-module.

Definition 3.1: Let $R$ be a fuzzy topological ring ,the set $E$ is said to be left fuzzy topological module on the fuzzy topological ring $\mathrm{R}$ if:

(1) E left fuzz module on R.

(2) $\mathrm{E}$ is a fuzzy topology compatible with the stricture of fuzzy group on $\mathrm{E}$ and satisfies the following axiom :

The mapping $\mathrm{R} \times \mathrm{E} \rightarrow \mathrm{E}$ defined by $(\lambda, \mathrm{x}) \rightarrow \lambda \mathrm{x}$ is a fuzzy continuous.

Proposition3.2 : If $E$ be an $R$-module over a ring $R,(E, \tau)$ and $(R, \delta)$ fuzzy topological spaces then $E$ is a left fuzzy topological module on $\mathrm{R}$ (left fuzzy topological R-module),If it satisfies the following conditions :-

(1) A function from $\mathrm{R} \times \mathrm{E} \rightarrow \mathrm{E} \quad,((\lambda, \mathrm{x}) \rightarrow \lambda \mathrm{x})$ is fuzzy continuous.

(2) A function from $\mathrm{R} \times \mathrm{R} \rightarrow \mathrm{R},((\mathrm{x}, \mathrm{y}) \rightarrow \mathrm{x}+\mathrm{y})$ is fuzzy continuous.

(3) A function from $\mathrm{R} \times \mathrm{R} \rightarrow \mathrm{R},((\mathrm{x}, \mathrm{y}) \rightarrow \mathrm{xy})$ is fuzzy continuous.

(4) A function from $\mathrm{E} \times \mathrm{E} \rightarrow \mathrm{E},((\mathrm{x}, \mathrm{y}) \rightarrow \mathrm{x}+\mathrm{y})$ is fuzzy continuous .

(5) A function from $\mathrm{E} \rightarrow \mathrm{E},\left(\mathrm{x} \rightarrow \mathrm{x}^{-1}\right)$ is fuzzy continuous .

Proof: It is follows directly from definition of fuzzy topological group ,fuzzy topological ring and fuzzy topological Rmodule.

From the Proposition above and the equivalent the concept of continuity we can write,

Proposition3.3 :If $E$ be an R-module over a ring $R,(E, \tau)$ and $(R, \delta)$ fuzzy topological spaces then $E$ is a left fuzzy topological module on $\mathrm{R}$ (left fuzzy topological R-module),If it satisfies the following conditions :-

(1) $\quad \forall(\lambda, x) \in R \times E$ and $\forall V$ nbh. of $(\lambda x)_{t}$ in $E, \exists U$ nbh. of $\lambda_{t}$ in $R$ and,$\exists W$ nbh. of $x_{t}$ in $E$ such that $U W \leq V$.

(2) $\quad \forall(\mathrm{x}, \mathrm{y}) \in \mathrm{R} \times \mathrm{R}$ and $\forall \mathrm{V}$ nbh. of $(\mathrm{x}+\mathrm{y})_{\mathrm{t}}$ in $\mathrm{R}, \exists \mathrm{U}$ nbh. of $\mathrm{x}_{\mathrm{t}}$ in $\mathrm{R}$ and,$\exists \mathrm{W}$ nbh. of $\mathrm{y}_{\mathrm{t}}$ in $\mathrm{R}$ such that $\mathrm{U}+\mathrm{W} \leq \mathrm{V}$.

(3) $\quad \forall(\mathrm{x}, \mathrm{y}) \in \mathrm{R} \times \mathrm{R}$ and $\forall \mathrm{V}$ nbh. of $(\mathrm{xy})_{\mathrm{t}}$ in $\mathrm{R}, \exists \mathrm{U}$ nbh. of $\mathrm{x}_{\mathrm{t}}$ in $\mathrm{R}$ and,$\exists \mathrm{W}$ nbh. of $\mathrm{y}_{\mathrm{t}}$ in $\mathrm{R}$ such that $\mathrm{UW} \leq \mathrm{V}$.

(4) $\quad \forall(\mathrm{x}, \mathrm{y}) \in \mathrm{E} \times \mathrm{E}$ and $\forall \mathrm{V}$ nbh. of $(\mathrm{xy})_{\mathrm{t}}$ in $\mathrm{E}, \exists \mathrm{U}$ nbh. of $\mathrm{x}_{\mathrm{t}}$ in $\mathrm{R}$ and,$\exists \mathrm{W}$ nbh. of $\mathrm{y}_{\mathrm{t}}$ in $\mathrm{E}$ such that $\mathrm{UW} \leq \mathrm{V}$.

(5) $\forall x \in E$ and $\forall V^{-1}$ nbh. of $x_{t}^{-1}$ in $E, \exists V$ nbh. of $x_{t}$ in $E$ such that $V \leq V^{-1}$.

Also, if we take fuzzy singleton point $0_{t}$ in above proposition we can write equivalent proposition.

Proposition3.4: Let R be a fuzzy left topological ring and E left fuzzy topological R-module. If $\omega$ the fuzzy nbh. base of $0_{\mathrm{t}}$ in $\mathrm{E}$ then $\omega$ satisfies the following conditions:

(1) $\forall V \in \omega, \exists W$ nbh. of $0_{t}$ in $R$ and $U \in \omega$ such that $W U \leq V$.

(2) $\quad \forall \mathrm{V} \in \omega, \exists \mathrm{U}, \mathrm{W} \in \omega$ such that $\mathrm{U}+\mathrm{W} \leq \mathrm{V}$.

(3) $\forall \mathrm{V} \in \omega, \exists U \in \omega$ such that $U \leq \mathrm{V}^{-1}$.

(4) $\forall V \in \omega$ and $\forall a_{\mathrm{t}} \in \mathrm{E}, \exists \mathrm{W}$ nbh. of $0_{\mathrm{t}}$ in $\mathrm{R}$, such that $\mathrm{Wa}_{\mathrm{t}} \leq \mathrm{V}$.

(5) $\forall V \in \omega$ and $\forall \mathrm{a}_{\mathrm{t}} \in \mathrm{R}, \exists \mathrm{U} \in \omega$, such thata $\mathrm{a}_{\mathrm{t}} \mathrm{U} \leq \mathrm{V}$.

In the opposite case, if $\omega$ is a filter base on $E$ satisfies the condition from 1 to 5 , then there exist unique fuzzy topology on E such that E left fuzzy topological R-module, and If $\omega$ the fuzzy nbh. base of $0_{t}$ in $E$.

Corollary3.5 :If $\omega$ is a filter base of left fuzzy topological submodules from fuzzy topological R-module E and R is a discrete fuzzy topological space, then $\omega$ is a nbh. base of $0_{t}$ in $E$.

Proposition 3.6 Let $E$ be a left fuzzy topological R-module. Then:

(1) $\forall \mathrm{a} \in \mathrm{E}$, the fuzzy translation $\mathrm{f}_{\mathrm{a}}(\mathrm{x})=\mathrm{x}+\mathrm{a}$ From fuzzy topological space $\mathrm{E}$ to itself is fuzzy homeomorphism.

(2) $\quad \forall \mathrm{a} \neq 0 \in \mathrm{R}$, the left translation $\mathrm{g}_{\mathrm{a}}(\mathrm{x})=\mathrm{a} \cdot \mathrm{x}$ From fuzzy topological space $\mathrm{E}$ to itself is fuzzy continuous.

(3) For all invertible element $b$, the left translation $g_{b}(x)=b$. $x$ From fuzzy topological space $E$ to itself is fuzzy homeomorphism.

Proof: (1) Suppose V any fuzzy nbh. of $(a+x)_{t}\left((a+x)_{t} \leq V\right)$ for any $t \in(0,1]$ by proposition above ,there exists fuzzy nbh. $\mathrm{U}$ of $\mathrm{a}_{\mathrm{t}}$ and fuzzy nbh. $\mathrm{W}$ of $\mathrm{x}_{\mathrm{t}}$ and $\mathrm{U}+\mathrm{W} \leq \mathrm{V}$, from the definition of $\mathrm{U}+\mathrm{W}$, and since $0<t \leq U(a)$ we have $(U+W)(y) \geq W(x)$. Also $f_{a}(W)(y)=\sup \left\{W(\alpha): f_{a}(\alpha)=y\right\}=\sup \{W(\alpha): a+\alpha=y\}=W(x)$.

Hence, $(\mathrm{U}+\mathrm{W})(\mathrm{y}) \geq \mathrm{f}_{\mathrm{a}}(\mathrm{W})(\mathrm{y}), \forall \mathrm{y} \in \mathrm{E}$. Therefore, $\mathrm{f}_{\mathrm{a}}(\mathrm{W}) \leq \mathrm{U}+\mathrm{W} \leq \mathrm{V}$,proving $\mathrm{f}_{\mathrm{a}}$ to be a fuzzy continuous, $\forall \mathrm{a} \in \mathrm{E}$. Defining $h_{a}: E \rightarrow E$ by $h_{a}(x)=-a+x$, we fined $h_{a}=f_{a}^{-1}$,i.e., $f_{a}$ is bijectve. Also,$h_{a}=f_{-a}$ and so $h_{a}$ is also fuzzy continuous. Hence, $f_{a}$ is a fuzzy homeomorphism.

(2) Suppose $V$ any fuzzy nbh. of $(a x)_{t}\left((a x)_{t} \leq V\right)$ for any $t \in(0,1]$ by proposition above ,there exists fuzzy $n b h$. $U$ of $a_{t}$ and fuzzy nbh. W of $x_{t}$ and $U W \leq V$, from the definition of $U W$, and since $0<t \leq U(a)$ we have $(\mathrm{UW})(\mathrm{y}) \geq \mathrm{W}(\mathrm{x})$. $\operatorname{Alsof}_{a}(W)(y)=\sup \left\{W(\alpha): f_{a}(\alpha)=y\right\}=\sup \{W(\alpha): a \alpha=y\}=W(x)$.

Hence, $(U W)(y) \geq \mathrm{f}_{\mathrm{a}}(\mathrm{W})(\mathrm{y}), \forall \mathrm{y} \in$ E. Therefore, $\mathrm{f}_{\mathrm{a}}(\mathrm{W}) \leq \mathrm{UW} \leq \mathrm{V}$, proving $\mathrm{f}_{\mathrm{a}}$ to be a fuzzy continuous. 
(3) Suppose $V$ any fuzzy nbh. of $(b x)_{t}\left((b x)_{t} \leq V\right)$ for any $t \in(0,1]$ by proposition above ,there exists fuzzy nbh. $U$ of $b_{t}$ and fuzzy nbh. $\mathrm{W}$ of $\mathrm{x}_{\mathrm{t}}$ and $\mathrm{UW} \leq \mathrm{V}$, from the definition of $\mathrm{UW}$, and since $0<t \leq U(b)$ we have (UW)(y) $\geq \mathrm{W}(\mathrm{x})$. Also $g_{b}(W)(y)=\sup \left\{W(\alpha): g_{b}(\alpha)=y\right\}=\sup \{W(\alpha): b \alpha=y\}=W(x)$.

Hence, $(\mathrm{UW})(\mathrm{y}) \geq \mathrm{g}_{\mathrm{b}}(\mathrm{W})(\mathrm{y}), \forall \mathrm{y} \in \mathrm{E}$. Therefore $\mathrm{g}_{\mathrm{b}}(\mathrm{W}) \leq \mathrm{UW} \leq \mathrm{V}$,proving $\mathrm{g}_{\mathrm{b}}$ to be a fuzzy continuous. Since $\mathrm{b}$ be an invertible element of $R$, we can Defining $h_{b}: E \rightarrow E$ by $h_{b}(x)=b^{-1} x$, we fined $h_{b}=g_{b}^{-1}$,i.e., $g_{b}$ is bijectve. Also, $h_{b}=$ $\mathrm{g}_{\mathrm{b}}{ }^{-1}$, and so $\mathrm{h}_{\mathrm{b}}$ is also fuzzy continuous. Hence, $\mathrm{g}_{\mathrm{b}}$ is a fuzzy homeomorphism.

Theorem 3.7 Let $\mathrm{R}$ be a left fuzzy topological module over topological ring $\mathrm{R}$, for each $\mathrm{t}$ with $0<t \leq 1$ and $\mathrm{x} \in$ E, then:

(1) If $V$ is fuzzy open in $E$, then $x_{t}+V$ is fuzzy open.

(2) If $V$ is fuzzy closed in $E$, then $x_{t}+V$ is fuzzy closed.

(3) If $\mathrm{V}$ is fuzzy open in $\mathrm{E}$ and $\mathrm{W}$ any fuzz set in $\mathrm{E}$, then $\mathrm{W}+\mathrm{V}$ is fuzzy open.

(4) If $\mathrm{V}$ is a fuzzy closed in $\mathrm{E}$ and $\mathrm{W}$ any closed set it has only finite elements its grading is non zero ,another elements its grading is zero, then $\mathrm{W}+\mathrm{V}$ is closed.

Proof.(1) Suppose V fuzzy open in E , since $f_{a}$ is a fuzzy homomorphism from E onto itself and therefore fuzzy open and fuzzy closed function. Now,

$\mathrm{f}_{\mathrm{a}}(\mathrm{V})(\mathrm{y})=\sup \left\{\mathrm{V}(\mathrm{x}): \mathrm{x}=\mathrm{f}_{\mathrm{a}}^{-1}(\mathrm{y})\right\}=\left(\mathrm{x}_{\mathrm{t}}+\mathrm{V}\right)(\mathrm{y})$, hence $\mathrm{x}_{\mathrm{t}}+\mathrm{V}$ open.

(2) Similar to (1)

(3) For each $a \in E$, let $W_{a}$ be fuzzy set in $E$ defined by $W_{a}(x)=\min \{W(a),(a+V)(x)\}=\min \{W(a), V(x-a)\}$ by translation each $\mathrm{W}_{\mathrm{a}}$ is open. Since $\mathrm{W}+\mathrm{V}=\sup _{\mathrm{a} \in \mathrm{E}} \mathrm{W}_{\mathrm{a}}$, the result follows.

(4) Since $W$ closed set it has only finite elements its grading is non zero, another elements its grading is zero, by (3) and the fact that the union of finite number of closed sets is closed, the result follows.

Theorem3.8[8]:Let $\left\{\left(\mathrm{X}_{\alpha}, \tau_{\alpha}\right)\right\}, \alpha=1,2, \ldots, \mathrm{n}$ be a finite family of compact fuzzy topological spaces. Then product fuzzy topological space $(X, \tau)$ is also compact.

Theorem3.9: Let A, B and C be fuzzy subsets of a left fuzzy topological

R-module E.

(1) If $A$ and $B$ are fuzzy compact, then $A B$ is fuzzy compact.

(2) If $\mathrm{C}$ is fuzzy compact, then $\mathrm{C}^{-1}$ is fuzzy compact.

Proof. (1) Let $\mathrm{f}: \mathrm{E} \times \mathrm{E} \rightarrow \mathrm{E}$ be a map defined by $\mathrm{f}(\mathrm{x}, \mathrm{y})=\mathrm{xy}$.

Then $\mathrm{f}$ is fuzzy continuous. By Theorem $3.8, \mathrm{~A} \times \mathrm{B}$ is compact.

Since the fuzzy continuous image of fuzzy compact set is fuzzy compact, $f(A, B)=f(A \times B)=A B$ is fuzzy compact.

(2) Let $f: E \rightarrow E$ be a map defined by $f(x)=x^{-1}$. Since $f$ is fuzzy continuous and the fuzzy continuous image of fuzzy compact set is fuzzy compact, $\mathrm{f}(\mathrm{C})=\mathrm{C}^{-1}$ is fuzzy compact.

Theorem3.10: Let E be a left fuzzy topological R-moule.Then $\mathrm{E}$ is fuzzy discrete module if and only if annihilator of all elements in $\mathrm{E}$ is fuzzy open ideal in $\mathrm{R}$.

Proof: by definition and fuzzy translation the prove immediately holds.

Definition3.11 : Let E be a left fuzzy topological R-moule, a fuzzy subset M from E is said to be left fuzzy topological Rsubmodule if:

(1) M left fuzzy R-submodule of E.

(2) M fuzzy subspace of fuzzy topological space E.

Proposition3.12 :If M a left fuzzy R-submodule from left fuzzy topological R-module E, then $M$ with relative fuzzy topology is a left fuzzy topological R-submodule.

Proof: from the definition 3.1 and the reduced any continuous function on subset of $M$ is continuous also, here we get any fuzzy R- submodule is a fuzzy topological R-submodule.

Theorem3.13: Let $\mathrm{E}$ be a left fuzzy topological R-module and let M be left fuzzy topological R-submodule, then closure $\mathrm{M}$ is a left fuzzy topological R-submodule.

Proof: We must be prove, if $a_{t} \in \bar{M}$ and $b_{t} \in \bar{M}$, then $(a-b)_{t} \in \bar{M}$ and if $r_{t} \in R$, then $(r a)_{t} \in \bar{M}$. Let W be a fuzzy nbh. of $\mathrm{a}-\mathrm{b}$ and let $\mathrm{U}, \mathrm{V}$ nbhs. of $\mathrm{a}$ and $\mathrm{b}$ respectively such that $\mathrm{U}-\mathrm{V} \leq \mathrm{W}$, there exists $\mathrm{x}, \mathrm{y}$ in $\mathrm{M}$ such that $\mathrm{x}_{\mathrm{t}} \in \mathrm{U}, \mathrm{y}_{\mathrm{t}} \in \mathrm{V}$, but $(x-y)_{t} \in M$ and $(x-y)_{t} \in W$,therefore $W \wedge M \neq 0_{t}$ and hence $(a-b)_{t} \in \bar{M}$. Similar let $W$ be a fuzzy nbh. of ra and let Ú,V́ nbhs. of $r$ and a respectively such that ÚV $\leq W$,there exists x́,ý in $M$ such that $x_{t} \in U$, ýt $\in \mathrm{V}$,but (xý) $)_{t} \in M$ and $\left(x^{\prime}\right)_{t} \in W$,therefore $W \wedge M \neq 0_{t}$ and hence $(\mathrm{ra})_{t} \in \bar{M}$, so $\bar{M}$ is a left fuzzy R-submodule of $E$ and hence $\bar{M}$ a left fuzzy topological R-submodule. 


\section{REFERENCES}

[1] Deb A. Ray, On (Left) Fuzzy Topolo- gical Ring, International Mathematical Forum, (2011), 6, 27,1303 - 1312.

[2] Lakshmana, V. GomathiNayagam, GeethaSivaraman, Induced topology on fuzzy singletons, Far East Journal of Applied Mathematics, ,(2008), 32 , 2189-198.

[ 3] Ma, J.L. C.H. Yu, Fuzzy topological groups, Fuzzy Sets andSystems, (1984) 12 ,289-299.

[4] Nanda,S.,"Fuzzy Modules over fuzzy rings”, Bull. Col.Math. Soc. (1989), 81, 197-200.

[5] Palaniappan, N. , Fuzzy Topology (Second Edition), Narosa Publishing House, India2005.

[6] Nayagam, V.L.G.D.Gauld,G.Venkateshwariand Sivaraman, "Strong Fuzzy Topological Group" New ZlandJournal of mathematics (2008),38, 187-195.

[7] Pu , P. M. and Y. M. Liu, Fuzzy topology I. Neighborhood structure of a fuzzy point and Moore-Smith convergence, J. Math. Anal. Appl(1980). 76, 571-599.

[8] Rosenfeld, A. ,Fuzzy groups, J.Math. Anal. Appl. (1971), 35,512-517.

[9] Zadeh, L.A.,"Fuzzy sets", Inf. Control,(1965 ), 8,338-353.

[10] ) Zheng Pan, Fu.,"Fuzzy Finitely generated Modules", Fuzzy sets and systems,(1987), 21, 105-113.

[11] Zheng Pan, Fu.,"Fuzzy Finitely Value Distribution of Fuzzy Vector spaces and fuzzy Modules, Fuzzy sets and systems," (1993),55, 319-322 